\title{
Survival of patients with a new diagnosis of heart failure: a population based study
}

\author{
M R Cowie, D A Wood, A J S Coats, S G Thompson, V Suresh, P A Poole-Wilson, \\ G C Sutton
}

\begin{abstract}
Objective-To describe the survival of a population based cohort of patients with incident (new) heart failure and the clinical features associated with mortality.

Design-A population based observational study.

Setting-Population of 151000 served by 82 general practitioners in west London.

Patients-New cases of heart failure were identified by daily surveillance of acute hospital admissions to the local district general hospital, and by general practitioner referral of all suspected new cases of heart failure to a rapid access clinic.

Interventions-All patients with suspected heart failure underwent clinical assessment, and chest radiography, ECG, and echocardiogram were performed. A panel of three cardiologists reviewed all the data and determined whether the definition of heart failure had been met. Patients were subsequently managed by the general practitioner in consultation with the local cardiologist or admitting physician.

Main outcome measures-Death, overall and from cardiovascular causes.

Results-There were 90 deaths (83 cardiovascular deaths) in the cohort of 220 patients with incident heart failure over a median follow up of 16 months. Survival was $81 \%$ at one month, $75 \%$ at three months, $70 \%$ at six months, $62 \%$ at 12 months, and $57 \%$ at 18 months. Lower systolic blood pressure, higher serum creatinine concentration, and greater extent of crackles on auscultation of the lungs were independently predictive of cardiovascular mortality (all $\mathrm{p}<0.001)$.

Conclusions-In patients with new heart failure, mortality is high in the first few weeks after diagnosis. Simple clinical features can identify a group of patients at especially high risk of death. (Heart 2000;83:505-510)
\end{abstract}

Keywords: heart failure; prognosis; survival; epidemiology

The prognosis of patients with a new diagnosis of heart failure in the United Kingdom is not known. Most of the published reports on the survival of patients with heart failure are based on randomised clinical trials of pharmacological treatment with agents such as angiotensin converting enzyme (ACE) inhibitors. ${ }^{12}$ The survival of such patients may not reflect the true picture for the generality of patients with heart failure because of the highly selected nature of patients entering clinical trials. Few reports have been published from population based studies. In Europe, data are limited to those from the study of men born in $1913,{ }^{3}$ with a reported survival of $74 \%$ at five years.In the USA, the survival of incident (new) cases of heart failure identified during thelast 40 years of follow up of the Framingham heart study cohort has been reported as $57 \%$ at one year and $25 \%$ at five years in men and $64 \%$ at one year and $38 \%$ at five years in women. ${ }^{4}$

There is controversy concerning which clinical factors identify patients at especially high risk of death. Most previous reports are based on data from randomised clinicaltrials, ${ }^{5}$ or from series of patients who survive for two years or more but remain severely symptomatic and are assessed for cardiac trans-plantation. ${ }^{67}$

The Hillingdon heart failure study was set up to determine the incidence and aetiology of heart failure within a general population. Over a 20 month period, 220 incident (new) cases of heart failure were identified from a population of 151000 individuals. Follow up of this cohort has provided an opportunity to describe the contemporary survival of this unselected group of patients with a new diagnosis of heart failure, and also to describe the associations between clinical features at presentation and survival.

\section{Methods}

The method of case identification has been described previously, ${ }^{8}$ but is summarised below.

STUDY POPULATION AND IDENTIFICATION OF CASES

The study population was that registered with 31 general practices (82 general practitioners) within the southern half of Hillingdon district in west London. The total study population was 151000 as of February 1996 (the midpoint of the period of identification of cases which ran from April 1995 to December 1996). The general practitioners agreed to refer all suspected cases of new heart failure to a rapid access clinic held at Hillingdon Hospital. Patients who were acutely ill were sent directly to the accident and emergency department of Hillingdon Hospital in the usual way and were identified by daily surveillance of all hospital admissions by a research nurse. From an audit of practice notes, it was estimated that 
Table 1 Clinical features of the 220 incident cases of heart failure

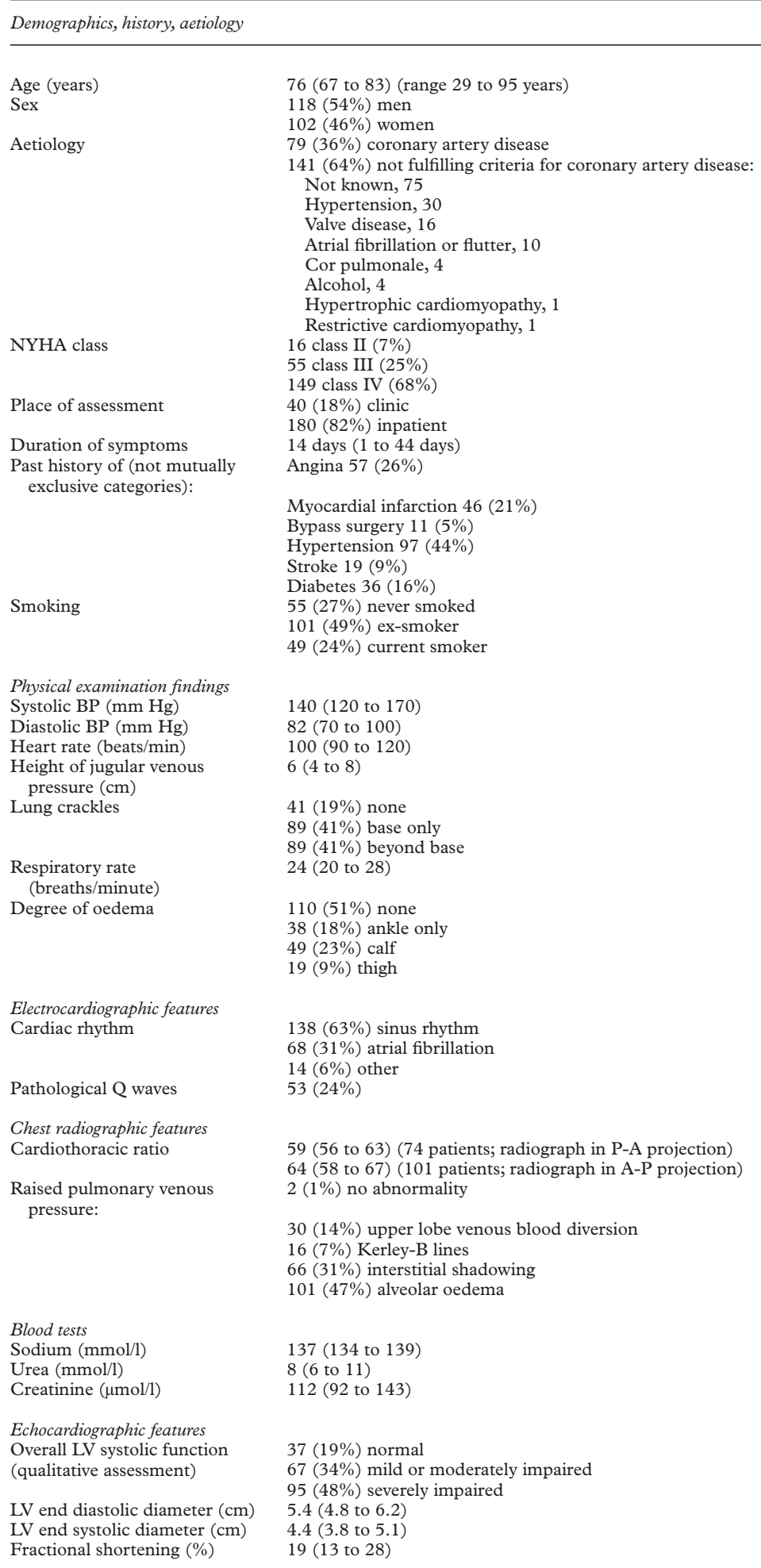

For continuous variables the numbers refer to median (interquartile range); for categorical variables the numbers refer to the number of cases (and percentage of the total cases for which the value of this variable is known).

BP, blood pressure; LV, left ventricular; NYHA, New York Heart Association; A-P, anterioposterior; $\mathrm{P}-\mathrm{A}$, posterioanterior. hospital admission. In such cases, the clinical findings of the admitting doctors were noted. Whenever possible an ECG, chest radiograph, transthoracic echocardiogram, serum biochemistry, haematology, and thyroid function tests were performed. The echocardiogram was done to a standard protocol and according to accepted guidelines ${ }^{910}$ by either one cardiologist (MRC) or one of two experienced cardiac technicians. The cross sectional, $M$ mode, Doppler, and colour flow images were recorded on videotape for subsequent analysis. Of the 220 new cases of heart failure identified, $217(99 \%)$ had an ECG, $216(98 \%)$ a chest radiograph, and 201 (91\%) an echocardiogram.

Approval for the study was granted by the local health authority ethics committee.

\section{CASE DEFINITION}

There is no gold standard for the diagnosis of heart failure, and there has been much variation in the diagnostic criteria used in previous studies. ${ }^{11}$ We adopted the criteria recommended by the working group on heart failure of the European Society of Cardiology. ${ }^{12}$ To meet the case definition of heart failure, patients had to have appropriate symptoms (shortness of breath or fatigue) with clinical signs of fluid retention (pulmonary or peripheral) in the presence of an underlying abnormality of cardiac structure or function. If an element of doubt remained, a beneficial response to treatment for heart failure (for example, a brisk diuresis accompanied by substantial improvement in breathlessness) was taken to confirm the diagnosis. All data collected were presented to a panel of three cardiologists (AC, GCS, and DAW) who determined on the basis of a majority decision whether the case definition had been met, and the aetiology. ${ }^{8}$ An underlying abnormality of cardiac structure or function was necessary to confirm a case of heart failure, but echocardiographic abnormalities were not sufficient in themselves to diagnose heart failure-patients had to satisfy the full case definition. The panel decision regarding whether the case definition had been met had good reproducibility (Cohen's $\kappa=0.67)$, as did the allocation of aetiology (Cohen's $\kappa=0.63$ ).

Patients' hospital and general practitioner notes were checked to ensure that only those without a previous history of heart failure were included in the study. If a patient had been assessed at another hospital in the past, the physician in charge of the patient's care at that hospital was contacted for details of the consultation and investigations performed.

\section{ALLOCATION OF AETIOLOGY}

Coronary artery disease was considered to be the primary cause if the patient had a documented history of myocardial infarction (acute or in the past), unstable angina pectoris, a history of stable angina supported by evidence of reversible myocardial ischaemia, or coronary artery disease confirmed at coronary angiography. Coronary angiography was performed at the discretion of the physician caring
$90 \%$ of new heart failure cases had been successfully identified in the study. ${ }^{8}$

A cardiologist (MRC or VS) took a standardised medical history and examined all the patients except those who died rapidly after 


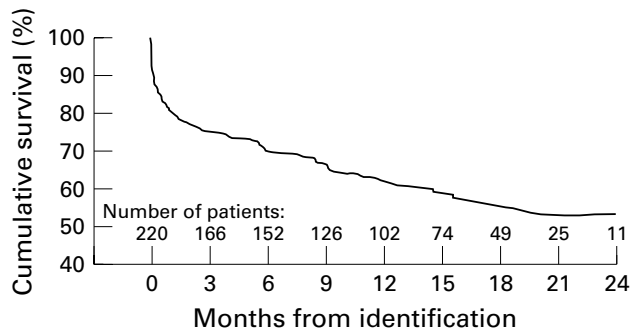

Figure 1 Survival of incident cases of heart failure.

Table 2 Survival in 220 patients with new heart failure, from time of diagnosis

\begin{tabular}{lll}
\hline Time (months) & Survival (\%) & $95 \%$ CI (\%) \\
\hline 1 & 81 & 76 to 87 \\
3 & 75 & 70 to 81 \\
6 & 70 & 64 to 76 \\
12 & 62 & 55 to 69 \\
18 & 57 & 50 to 64 \\
\hline
\end{tabular}

CI, confidence interval.

for the patient. Hypertension was considered to be the cause if there was a history of hypertension from the general practice records or sustained hypertension (blood pressure greater than $160 / 95 \mathrm{~mm} \mathrm{Hg}$ ) during hospital admission, and there was no documented history of myocardial infarction or angina, and no evidence of other cardiac pathology. The presence and severity of underlying valve heart disease was assessed from the history, clinical examination, and echocardiographic findings.

FOLLOW UP

Date of death and certified cause of death ${ }^{13}$ were identified by flagging each patient's record at the NHS Central Registry using their NHS number. Follow up for death was complete for all 220 cases to June 1997.
DATA ANALYSIS

Survival was estimated using the method of Kaplan-Meier. The standardised mortality ratio (SMR) for cardiovascular death was calculated by dividing the number of observed deaths from cardiovascular causes by the number of deaths expected if the appropriate national age and sex specific death rates were applied. ${ }^{14}$ The associations between clinical and investigative features and observed cardiovascular mortality were examined by Cox proportional hazards modelling, using the strategy recommended by Collett. ${ }^{15}$ All of the covariates associated with survival in the Cox univariate regression with $\mathrm{p}<0.15$ were initially entered into the model, and discarded one by one if they did not significantly $(\mathrm{p}<0.05)$ improve the fit of the model. Those who died of non-cardiovascular causes were censored at the time of death. Two tailed tests of significance are reported.

\section{Results}

Table 1 gives the basic features of the cases identified. During the study period, 220 new cases of heart failure were identified within the study population (118 male, 102 female; age range 29 to 95 years); 180 cases were identified from surveillance of admissions to the local hospital and the remaining 40 from 157 referrals to the rapid access clinic. The single most common cause was coronary heart disease (79 cases $(36 \%))$. The majority of cases were symptomatic at rest or on mild exertion (New York Heart Association functional class IV and III, respectively). A past history of cardiovascular disease was common and almost three quarters reported smoking at some time in their life. The physical examination, chest radiography, and echocardiographic features

Table 3 Results of univariate Cox proportional hazards modelling of cardiovascular mortality

\begin{tabular}{|c|c|c|c|c|c|c|c|}
\hline Variable & Units/coding & Number & $\begin{array}{l}\text { Hazard } \\
\text { ratio }\end{array}$ & $95 \% C I$ & $\chi^{2}(L R T)$ & $d f$ & $p$ Value \\
\hline Age (years) & 10 & 220 & 1.48 & 1.10 to 1.79 & 12.0 & 1 & $<0.001$ \\
\hline Sex & $\begin{array}{l}1=\text { male } \\
2=\text { female }\end{array}$ & 220 & 1.14 & 0.74 to 1.75 & 0.347 & 1 & 0.56 \\
\hline Smoking & $\begin{array}{l}1=\text { never } \\
2=\text { ex-smoker } \\
3=\text { current smoker }\end{array}$ & 205 & 1.31 & 0.96 to 1.79 & 2.87 & 1 & 0.09 \\
\hline Aetiology & CAD $v$ other & 220 & 1.45 & 0.93 to 2.22 & 2.61 & 1 & 0.11 \\
\hline NYHA class & II to IV & 220 & 1.95 & 1.26 to 3.03 & 11.0 & 1 & $<0.001$ \\
\hline Place of assessment & Inpatient $v$ clinic & 220 & 4.70 & 1.90 to 11.63 & 17.8 & 1 & $<0.001$ \\
\hline SBP & $10 \mathrm{~mm} \mathrm{Hg}$ & 220 & 0.91 & 0.85 to 0.97 & 8.53 & 1 & 0.004 \\
\hline DBP & $10 \mathrm{~mm} \mathrm{Hg}$ & 220 & 0.85 & 0.75 to 0.97 & 6.55 & 1 & 0.01 \\
\hline Crackles & $\begin{array}{l}0=\text { none } \\
1=\text { basal } \\
2=\text { more than basal }\end{array}$ & 219 & 2.25 & 1.61 to 3.15 & 25.6 & 1 & $<0.001$ \\
\hline ECG rhythm & $\begin{array}{l}\text { Sinus rhythm } v \text { atrial } \\
\text { fibrillation }\end{array}$ & 217 & 1.65 & 1.00 to 2.73 & 4.11 & 1 & 0.04 \\
\hline $\begin{array}{l}\text { Cardiothoracic ratio } \\
\text { (AP projection) }\end{array}$ & $10 \%$ & 101 & 1.78 & 1.18 to 2.69 & 7.48 & 1 & 0.006 \\
\hline Pulmonary congestion & $\begin{array}{l}\text { Score } 1 \text { (none) to } 5 \\
\text { (alveolar oedema) }\end{array}$ & 215 & 1.31 & 1.03 to 1.66 & 5.61 & 1 & 0.02 \\
\hline Sodium $(\mathrm{mmol} / \mathrm{l})$ & & 218 & 0.93 & 0.89 to 0.98 & 6.28 & 1 & 0.01 \\
\hline Urea $\left(\mathrm{mmol} / 1[\mathrm{log}]^{\star}\right)$ & & 218 & 1.47 & 1.16 to 1.85 & 10.5 & 1 & 0.001 \\
\hline Creatinine $\left(\mathrm{mol} / 1[\mathrm{log}]^{\star}\right)$ & & 218 & 2.56 & 1.88 to 3.47 & 29.9 & 1 & $<0.001$ \\
\hline LV systolic function & Impaired $v$ normal & 199 & 0.80 & 0.43 to 1.47 & 0.61 & 1 & 0.43 \\
\hline FS (\%) & & 80 & 1.03 & 0.99 to 1.08 & 1.99 & 1 & 0.19 \\
\hline LVEDD (cm) & & 102 & 0.77 & 0.51 to 1.16 & 1.56 & 1 & 0.21 \\
\hline $\operatorname{LVESD}(\mathrm{cm})$ & & 102 & 0.72 & 0.50 to 1.04 & 3.08 & 1 & 0.08 \\
\hline
\end{tabular}

*Hazard ratio for these variables refers to the ratio of hazard for a doubling in the serum concentration.

$\mathrm{AF}$, atrial fibrillation; CAD, coronary artery disease; CI, confidence interval; DBP, diastolic blood pressure; df, degrees of freedom; FS, fractional shortening; LRT, likelihood ratio test; LV, left ventricular; LVEDD, left ventricular end diastolic diameter; LVESD, left ventricular end systolic diameter; SBP, systolic blood pressure; SR, sinus rhythm. 
Table 4 Final model selected by multivariate Cox proportional hazards modelling as simplest description of cardiovascular mortality of the cohort of patients

\begin{tabular}{llllcc}
\hline Variable & Units/coding & Hazard ratio & $95 \%$ CI & $\chi^{2}$ (LRT) & p Value \\
\hline Age & $\begin{array}{l}10 \text { years } \\
\text { Crackles }\end{array}$ & 1.26 & 1.01 to 1.57 & 4.31 & 0.04 \\
& $\begin{array}{l}1=\text { basal } \\
2=\text { more than basal }\end{array}$ & 2.13 & 1.50 to 3.02 & 20.5 & $<0.001$ \\
SBP & $\begin{array}{l}10 \mathrm{~mm} \mathrm{Hg} \\
\text { Creatinine }\end{array}$ & 0.88 & & & \\
& $\mu \mathrm{mol}(\log )^{\star}$ & 2.64 & 0.83 to 0.94 & 17.7 & $<0.001$ \\
\end{tabular}

Creatinine values were log transformed before entry into the model.

${ }^{\star}$ Hazard ratio for a doubling in serum creatinine concentration.

CI, confidence interval; LRT, likelihood ratio test; SBP, systolic blood pressure.

were as would be expected in patients with heart failure. Almost $40 \%$ were not in sinus rhythm at presentation, and almost a quarter had ECG evidence of previous infarction (pathological Q wave).

SURVIVAL

Of the 220 patients included in the study, 90 died before the end of the follow up period, the mean follow up being 16 months (range 6-26 months). The majority of deaths were related to cardiovascular disease $(83(92 \%))$. Figure 1 depicts the survival curve for this cohort of patients. The survival was $81 \%$ at one month, $75 \%$ at three months, $70 \%$ at six months, $62 \%$ at 12 months, and $57 \%$ at 18 months (table 2). The SMR for cardiovascular deaths within the cohort was 18.1 at 12 months, representing an 18 -fold increase (95\% confidence interval (CI) 15-fold to 23-fold) compared with individuals of the same age and sex in the general population.

\section{CLINICAL FEATURES ASSOCIATED WITH}

\section{CARDIOVASCULAR MORTALITY}

On univariate Cox modelling, various clinical features were associated with cardiovascular death during the follow up period (table 3): increasing age, functional class, extent of lung crackles on auscultation, increasing cardiothoracic ratio and degree of pulmonary congestion on chest radiograph, higher serum urea or creatinine, lower serum sodium, lower blood pressure, and sinus rhythm as opposed to atrial fibrillation. There was weak evidence that the prognosis was worse if the aetiology was coronary artery disease compared with other causes $(\mathrm{p}=0.11)$, but this issue needs to be addressed in a larger cohort of heart failure cases. There was no evidence that sex or overall left ventricular systolic function (as assessed qualitatively or by fractional shortening) was associated with survival.

Many of the clinical features are closely related. On multivariate Cox modelling only four features were found to be independently associated with death: age, blood pressure, serum creatinine concentration, and the extent of lung crackles on auscultation (table 4).

\section{Discussion}

SURVIVAL FROM HEART FAILURE

The survival of incident (new) cases of heart failure identified in this study is poor: more than a third of patients had died within 12 months of the diagnosis. This is considerably worse than the prognosis reported from recent drug trials recruiting patients with mild to moderate symptoms. The SOLVD study (studies of left ventricular dysfunction) reported mortality at only $12 \%$ at one year in the active treatment arm. ${ }^{16}$ In a recently published trial of $\beta$ blockade in patients with heart failure the mortality in the group not given bisoprolol was only $13 \%$ at one year. ${ }^{17}$

There are several possible explanations for such a discrepancy. First, clinical trials tend to recruit a select group of patients with a better prognosis than the generality of patients with heart failure. The typical average age of patients in a drug trial for heart failure is 60 years, compared with around 75 years for the generality of patients with heart failure. ${ }^{411}$ Most trials also require that a patient has had stable heart failure for some months before recruitment; the average duration of heart failure for many patients entering clinical trials is two or three years. Such patients are "natural survivors" in the sense that they have survived the early high risk period. Heart failure is often associated with considerable comorbidity, but such patients are also unlikely to be recruited to clinical trials. These biases will make the prognosis of heart failure appear better in clinical trials than it actually is for most patients. Second, the case definition employed in this study was for clinical heart failure, requiring firm evidence of appropriate symptoms, underlying cardiac dysfunction, and fluid retention. This may be reflected in a worse prognosis than if a less strict definition was employed.

Our results, however, are very similar to those reported from population based studies in the USA - the Framingham heart study, ${ }^{4}$ the Rochester epidemiology project, ${ }^{18}$ and the Olmsted County study. ${ }^{19}$ These studies also identified incident cases of heart failure in the general population, but used a diagnostic system based on a heart failure "score" rather than a clinical definition as employed in this study. ${ }^{11}$ The only European data with which our results can be compared come from a cohort of men born in Gothenburg and initially aged 50 years who were followed up for 17 years. ${ }^{3}$ The case definition was much less stringent and led to a diagnosis of heart failure much more often than would be the case in clinical practice. ${ }^{20}$ The five year survival $(74 \%)$ was much better than found in this study or in the population based studies from the USA, but this is not surprising considering the loose case definition and the limited and younger age range of the individuals identified.

CLINICAL FEATURES ASSOCIATED WITH DEATH Most of the published reports on the clinical features predicting a poor outcome in patients with heart failure have come from highly selected series of patients. Typically, series are limited to young patients who have been referred for cardiac transplant assessment or for the introduction of vasodilator treatment. Such patients have survived the initial onset of heart failure, but remain severely symptomatic despite pharmacological treatment at their local hospital. ${ }^{6}{ }^{21-30}$ These patients are unrepresentative of all patients with heart failure. 
Also, such patients tend to undergo investigations that are not readily available for patients with heart failure in routine clinical practice (for example, cardiac catheterisation, exercise testing with gas exchange analysis). The prognostic scoring systems derived from such data are unlikely to be applicable to the generality of patients with heart failure and have little use in clinical practice.

In our present study four factors were found to be independently associated with cardiovascular mortality: age, serum creatinine concentration, systolic blood pressure, and the extent of crackles on auscultation of the lungs.

Mortality increased by $26 \%$ for every 10 year increase in age at onset of heart failure, even when allowing for other factors (table 4). Although intuitively this might be expected, and other investigators have found it to be so, ${ }^{22} 3132$ there are reports suggesting the opposite $^{33}$ or no association at all. ${ }^{24}$

Serum creatinine concentration has previously been reported to be associated with prognosis in a series of 203 patients referred for stabilisation of heart failure. ${ }^{30}$ In that report, the serum sodium and urea concentrations were also associated with prognosis, as has been confirmed by others ${ }^{27}$ and by the univariate analyses in our study. Patients with heart failure and a low serum sodium concentration or high urea and creatinine have a higher serum renin concentration, suggesting that serum creatinine may be taken as a marker of reduced cardiac output and renal perfusion and consequent activation of the renin-angiotensin axis. Such patients are likely to be clinically decompensated and to have high circulating concentrations of catecholamines and a limited ability to respond to situations or interventions that decrease cardiac output. ${ }^{34}$

Similarly, a higher systemic arterial pressure has also been reported to be associated with better survival in patients with heart failure. ${ }^{766^{35}{ }^{36}}$ It is rather surprising that a single blood pressure measurement is so strongly associated with survival, but it may reflect cardiac function or, more probably, the ability of the circulatory system to generate adequate perfusion pressure despite a low cardiac output.

The extent of lung crackles at presentation was strongly related to subsequent prognosis. This is despite the fact that such crackles are poor markers of raised left ventricular filling pressures in chronic heart failure. ${ }^{37}$ In this study no invasive measurements were made and thus it is impossible to determine whether this is also the case for incident heart failure. It is possible that the increase in lymphatic drainage found in the lungs in chronic heart failure has not had time to occur in patients whose symptoms have been present for a much shorter period of time. In such circumstances, the extent of lung crackles may be a better indicator of cardiac function. Some evidence for this is provided by the strong association found in this study between the extent of lung crackles and the degree of pulmonary congestion on a chest radiograph (Mantel-Haenszel test for linear association, $\mathrm{p}<0.001)$.
The variables found in our study to be independently associated with survival presumably reflect the degree of underlying cardiac dysfunction. More direct measures of cardiac dysfunction such as measurement of cardiac output at rest (and on exercise) were not available. No association was found between left ventricular systolic function (as assessed on echocardiography either qualitatively or quantitatively by fractional shortening) and survival. The dimensions of the left ventricle were measurable on $\mathrm{M}$ mode echocardiography in 102 cases (46\%). There was only weak evidence that the diameter of the left ventricle at end systole was associated with survival (table 3). Our inability to find an association between ventricular systolic function so assessed and prognosis may reflect a lack of statistical power because of small numbers, or the limited information on overall cardiac and circulatory function provided by such measurements. In this study, only new cases of heart failure were identified, and the chronic process of remodelling and ventricular dilatation may not have had time to occur. Previous reports, however, have not always shown an association between left ventricular systolic function as assessed by ejection fraction and survival in patients with heart failure..$^{38-40}$

\section{CONFOUNDING BY TREATMENT}

After identification as a new case of heart failure in this study, patients were not managed according to a specific protocol. The management was determined by the admitting physician and subsequently by the general practitioner in consultation with the hospital physician. It is therefore possible that the association between the four clinical features in table 4 and survival could be explained by the confounding effect of treatment prescribed. This would arise if the treatment had an effect on survival and if the decision to prescribe the treatment was influenced by these clinical features. This is certainly a consideration for ACE inhibitors, which improve the prognosis of heart failure ${ }^{16}$ and may be more likely to be prescribed in younger patients, those with a higher blood pressure, and those with less elevated serum creatinine concentration. In this study data were available on whether ACE inhibitors had been prescribed for 163 of the 179 patients surviving more than 30 days. Of these, $106(65 \%)$ were prescribed an ACE inhibitor. ACE inhibitors were significantly less likely to be prescribed in older patients $(p=0.01)$ and in patients with a higher serum creatinine concentration $(p=0.06)$. Adding a "treatment with ACE inhibitor" variable to the Cox multivariate analysis did not, however, appreciably change the magnitude of association between survival and age, systolic blood pressure, serum creatinine, or the extent of lung crackles. It is therefore unlikely that the associations described in table 4 are caused by confounding from the prescription of ACE inhibitors. 
CONCLUSIONS

This study has provided the first data in the UK on the prognosis of heart failure in an unselected cohort of incident cases of heart failure arising in the adult population. Survival was poor and many times worse than that of the general population. Simple clinical featuressuch as age, systolic blood pressure, serum creatinine concentration, and the extent of lung crackles on auscultation at presentation-were strongly associated with subsequent survival. Such features may allow clinicians to identify those patients with an especially poor prognosis.

This study was undertaken by the Clinical Epidemiology Group in Cardiac Medicine with grant support from the British Heart Foundation and the Wellcome Trust (MR Cowie). We thank Foundation and the Wellcome Trust (MR Cowie). We thank
Helen Penston (research nurse, Hillingdon Hospital), Alison Helen Penston (research nurse, Hillingdon Hospital), Alison
Rochelle and Christine Harrison (cardiac technicians, Hillingdon Hospital), Neil McLennan (data manager, Clinical Epidemiology Group) and Jane Ingham (research administrator miology Group) and Jane Ingham (research administrator, Clinical Epidemiology Group), and the patients and general practitioners who made this study possible. The audit of case registration data were kindly provided by the Office of National Statistics.

1 The CONSENSUS Trial Study Group. Effects of enalapril on mortality in severe congestive heart failure: results of the cooperative north Scandinavian enalapril survival study (CONSENSUS). N Engl f Med 1987;316:1429-35.

2 The SOLVD Investigators. Effect of enalapril on survival in patients with reduced left ventricular ejection fractions and congestive heart failure. N Engl f Med 1991;325:293-302.

3 Eriksson $\mathrm{H}$. Heart failure: a growing public health problem. f Intern Med 1995;237:135-41.

4 Ho KK, Pinsky JL, Kannel WB, et al. The epidemiology of heart failure: the Framingham study. $\mathcal{F}$ Am Coll Cardiol 1993;22:6-13A.

5 Cohn JN, Johnson GR, Shabetai R, et al. Ejection fraction, peak exercise oxygen consumption, cardiothoracic ratio, peak exercise oxygen consumption, cardiothoracic ratio,
ventricular arrhythmias, and plasma norepinephrine as ventricular arrhythmias, and plasma norepinephrine as
determinants of prognosis in heart failure. Circulation determinants of

6 Campana C, Gavazzi A, Berzuini C, et al. Predictors of prognosis in patients awaiting heart transplantation. $\mathcal{F}$ Heart Lung Transplant 1993;12:756-65.

7 Aaronson KD, Schwartz JS, Chen T-M, et al. Development and prospective validation of a clinical index to predict survival in ambulatory patients referred for cardiac transplan evaluation. Circulation 1997;95:2660-7.

8 Cowie MR, Wood DA, Coats A, et al. Incidence and aetiology of heart failure: a population-based study. Eur Heart f 1999;20:421-8.

9 Sahn DJ, DeMaria A, Kisslo J, et al. The committee on M-mode standardization of the American Society of Echocardiography. Recommendations regarding quantitation in M-mode echocardiographic measurements. Circulation 1978;58:1072-82

10 Henry WL, DeMaria A, Gramiak R, et al. Report of the American Society of Echocardiography committee on American Society of Echocardiography committee on cardiography. Circulation 1980; 62:212-17.

11 Cowie MR, Mosterd A, Wood DA, et al. The epidemiology of heart failure. Eur Heart f 1997;18:208-15.

12 The Task Force on Heart Failure of the European Society of Cardiology. Guidelines for the diagnosis of heart failure. Eur Heart $\mathcal{F}$ 1995;16:741-51.

13 World Health Organisation. Manual of the international statistical classification of diseases, injuries and causes of death. Geneva: WHO, 1977

14 Office for National Statistics. Mortality statistics: cause. Review of the Registrar General on deaths by cause, sex and age, in England and Wales, 1995. DH2 No 22. London: HMSO, 1997:176-9.
15 Collett D. Modelling survival data in medical research. London: Chapman and Hall, 1994.

16 The SOLVD Investigators. Effect of enalapril on survival in patients with reduced left ventricular ejection fractions and congestive heart failure. N Engl f Med 1991;325:293-302.

17 CIBIS-II Investigators and Committee. The cardiac insufficiency bisoprolol study II (CIBIS-II): a randomised trial. Lancet 1999;353:9-13.

18 Rodeheffer RJ, Jacobsen SJ, Gersh BJ, et al. The incidence and prevalence of congestive heart failure in Rochester, Minnesota. Mayo Clin Proc 1993;68:1143-50.

19 Senni M, Tribouilloy CM, Rodeheffer RJ, et al. Congestive heart failure in the community: a study of all incident cases in Olmsted County, Minnesota, in 1991. Circulation 1998; 98:2282-9.

20 Eriksson H, Caidahl K, Larsson B, et al. Cardiac and pulmonary causes of dyspnoea - validation of a scoring test for clinical-epidemiological use: the study of men born in 1913. Eur Heart f 1987;8:1007-14.

21 Massie BM, Conway M. Survival of patients with congestive heart failure: past, present, and future prospects. Circulation 1987;75:11-19

22 Fuster V, Gersh BJ, Giuliani ER, et al. The natural history of idiopathic dilated cardiomyopathy. Am F Cardiol 1981;47: 525-31

23 Lee TH, Hamilton MA, Stevenson LW, et al. Impact of left ventricular cavity size on survival in advanced heart failure. Am 7 Cardiol 1993;76:672-6.

24 Unverferth DV, Magorien RD, Moeschberger ML, et al. Factors influencing the one-year mortality of dilated cardiomyopathy. Am f Cardiol 1984;54:147-52.

25 Szlachcic J, Massie BM, Kramer BL, et al. Correlates and prognostic implication of exercise capacity in chronic congestive heart failure. Am f Cardiol 1985;55:1037-42.

26 Kelly TL, Cremo R, Nielsen C, et al. Prediction of outcome in late-stage cardiomyopathy. Am Heart f 1990;119:111121.

27 Parameshwar J, Keegan J, Sparrow J, et al. Predictors of prognosis in severe chronic heart failure. Am Heart f 1992; 123:421-6.

28 Scrutinio D, Lagioia R, Ricci A, et al. Prediction of mortality in mild to moderately symptomatic patients with left ventricular dysfunction. Eur Heart f 1994;15:1089-95.

29 Middlekauff HR, Stevenson WG, Stevenson LW. Prognostic significance of atrial fibrillation in advanced heart failure. A study of 390 patients. Circulation 1991;84:40-8.

30 Lee WH, Packer M. Prognostic importance of serum sodium concentration and its modification by converting enzyme inhibition in patients with severe chronic heart failure. Circulation 1986;73:257-67.

31 Johnson RA, Palacios I. Dilated cardiomyopathies of the adult. N Engl F Med 1982;307:1051-8.

32 Demakis JG, Proskey A, Rahimtoola SH, et al. The natural course of alcoholic cardiomyopathy. Ann Intern Med 1974; 80:293-7.

33 Kuhn H, Becker R, Fischer J, et al. Studies on the etiology, the clinical course and the prognosis of patients with dilated cardiomyopathy (DCM). Z Kardiol 1982;71:497508.

34 Dzau VJ, Packer M, Lilly LS, et al. Prostaglandins in severe congestive heart failure: relation to activation of the reninangiotensin system and hyponatremia. N Engl f Med 1984; 310:347-52.

35 Hatle L, Orjavik O, Storstein O. Chronic myocardial disease. I. Clinical picture related to long-term prognosis. Acta Med Scand 1976;199:399-405.

36 Cleland JGF, Dargie HJ, Ford I. Mortality in heart failure: clinical variables of prognostic value. Br Heart $\mathcal{F}$ 1987;58: $572-82$.

37 Stevenson LW, Perloff JK. The limited reliability of physical signs for estimating hemodynamics in chronic heart failure. fAMA 1989;261:884-8.

38 Kelly TL, Cremo R, Nielsen C, et al. Prediction of outcome in late-stage cardiomyopathy. Am Heart $\mathcal{F}$ 1990;119:111121 .

39 Lee WH, Packer M. Prognostic importance of serum sodium concentration and its modification by converting enzyme inhibition in patients with severe chronic heart failure. Circulation 1986;73:257-67.

40 Cowburn PJ, Cleland JGF, Coats AJS, et al. Risk stratification in chronic heart failure. Eur Heart $\mathcal{F}$ 1998;19:696-70. 